\title{
NOUVEAUX DISPOSITIFS POUR INTERDIRE AUX POISSONS L'ACGÈS DES DÉRIVATIONS HYDRAULIQUES
}

\author{
Par M. C. GAI.LOIS \\ Conservateur des Eaux et Forèts en retraitc, \\ et M. DE DROUIN DE BOUVILLE \\ Inspecteur principal des Eaux et Forêts en retraite.
}

Lorsque les Poissons qui, pour une cause ou une autre, dévalent un cours d'eau, abandonnant la coulière pour s'engager dans une dérivation, is courent des risques plus ou moins graves.

Dans les rigoles d'irrigation leur perte est quasi fatale; une fois parvenus aux artérioles du réseau, ils y reslent à sec quand le courant d'abreuvage et interrompu. Et s'il s'agit de canaux usiniers, c'est le passage par 'es coursiers des rones ou les ditributeurs des turbines.

A s'en référer à de récents travaux, cefte traversée des récepteurs hydrauliques serait moins dangereuse qu'on ne limagine. Les recherches de MM. Lundbeck (i), Schmassmaxy (2), Kneitmanx (3) ont montré que, sauf pour l'Anguille, le sectionnement tait plutôt l'exception que la règle (4). Des expériences d'une Commission d'experts badois et suisses, il résulte qu'à l'usine d'Augst, sur le Rhin, en amont de Bâle, ont pu sortir sans lésions, d'une turbine "Francis" de I m. 5o de diamètre, virant à 107 tours par minute et développant i.25o chevaux-vapeur : un Barbeau de 38 centimètres, une Brême de $20 \mathrm{~cm}$. 1/2, un Chevaine de 3 I cm. s/a, un Chondrostome de $29 \mathrm{~cm}$. I/2 et un Rotengle de $22 \mathrm{~cm}$. I/2. J'autre part, des Truites arc-en-ciel ont été capturées dans la rivière d'Ain, en aval de ?'usine de Marignier (Jura) qui, provenant certainement d'un repeuplement effectué dans le lac Chalain, avaient, en conséfquence, dù passer par la turbine. Le calcul a montré que cette dernière pouvait ne pas porter préjudice à des spécimens de 56 centimètres de longrueur et que, sur

(1) Vinlersuchungen äber die lieschadigung von Fischen, bcsondere: Aden, in den Turbinen des Krafluerlis Friedlatd. - Zeitschrift für Fischerei. Neumann, Neudamm, $\mathrm{XXV}, 3, \mathbf{1 9 2 7}$.

(3) V'ersuche äber dir Beschädigung ion Fischen durch Turbinen. - Schweizerische Fischerei Zeitung. Berne, XXXVI, I I Novembre $: 9^{28}$.

(3) Le franchissement des installations hydroélectriạues par le Poisson ; - Annales du Ministère de l'Agriculture, Paris, Imprimerie nalionale. Fis. I.VIII, p. 65, rg3o.

(f) Voir Bulletin, Juin 1929, p. 298 . 
100 sujets de ro centimètres, 9.3 devaient la franchir sans dommage. (I)

Mentionnons, en outre, la démonstration faite par M. Knerrmaxix que le Poisson est extraordinairement résistant aux choes physiologiques provoqués par des variations brusques de pression; il subit, sans paraître incommodé, en présence de l’air. des détentes de l'ordre de 20 atmosphères.

Il n'en reste pas moins que la traversée d'une turbine est toujours fatale aux Poissons dépassant une rertaino dimension, variable avec les caracléristiques de la machine, el, qu'all-dessous, il est d'aulant plus exposé à coincement entre une aube directrice el une autre réceptrice que sa lon:rueur est plus grande.

On conçoit donc la néressité d interdire, aux Poissons, l'accès des dérivalions quelles qu'elles soient.

Il semble qu'on les doive retenir facilement par l’installation, aux prises d'eau, de grilles ou de grrillages. I.e malheir est que les obstacles de ac śenre, si ingénieusement agencés que soient rertains d'entre eux, provoyuent un remous qui va s'arcenfuant au fur ef à mesure qu ils sont davantage obstrués par les herbes flottantes, les détritus charriés, etc. Dans ces conditions, il y a réduction du débit emprunté et, surtout, réduction sujette à variations fort amples el par l⿳亠 fort gênaute. On y remédie, dans une certaine mesure, par des nettoyages fréquents; mais ils sont toujours onéreux et volonticrs difficultueux.

Il était désirable quion trouvàt antro chose et le problème, qui ne laissdit pas d'être ardu, consistait à arriter le Poisson sans faire obstacle au courant. On doit, aujourd'hui, le considérer comme résolu, grâce aux recherches poursuivies res dernières anmécs aux Ftats-linis et dont les comptes rendus ont été récemment publiés par M. Elmer Higgrass (2), chargé du service des recherches scientifiques au Bureau of Fisheries de Washington (D. C.).

Depuis longtemps, dans les Élats du Nord-Ouest, on se plaignait des deshuctions massives de saumoneaux (3) descendant à la mer dans les dérisations établies pour les besoins de l'agriculture ou de l'industrie. D'au-

(1) La longueur maxima L du Poisson qui peut traverser unc turbine sans êlre lésé est dounée par la formule ci-apriss, ctablie par M. KherTmavi.

$$
\mathrm{I}==2-r \frac{V^{\circ}}{\mathrm{V}}\left(\frac{1}{n}-\frac{1}{n^{\prime}}\right)
$$

$r$ : rilyon de la circonférence extérieure du rotor ; - lo : vileste diultroduction de l ean dins la couronne mobile ou rotor : - V : vitesse dintrainement de cette conronne a sa circonférence extérieure; - 11 : nombre dautes de celfr couronne ; -.u' : jombre d'aubes du distributeur ou stalor.

(2) Elmer Higgas. - progress in biological imfuirics. I9:8; - inmexe $\mathrm{X}$ au Reporl of Commissioner of Fisheries for the fiscal vear 19:9, 1. 618. -- Washington, 193o.

progress in biological inquiries, 1920 ; -- Annexe $\mathrm{X} 1$ au Repert of Commissioner of Fisheries for the fiscel year 1930, p. logt. -- Washington, $19^{3}$.

(3) Il s'agrit de jeunes sujets de saumons du Pacifique (genre Oncorhynchaul ou de la

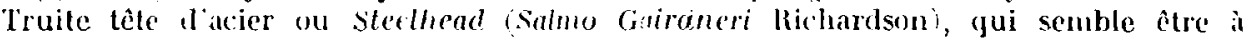
l'Are-en-ciel ce qu'est notre Truble de ener it lat Truile commune. 
Ires Poissons, effectuant ces déplacements saisonniers dont sunt coutumières beaucoup d'espèces, périssaient aussi en plus on moins grande abondance.

La situation devenant critique, une conférence d agents des Services de pèche du Gouvernement fédéral el des Ftats d'Idaho, Montana, Gregon et Washington, avec participation de représentants des intérèts commerciaux .t sportifs, eut lieu en Janvier 1928, à Yakima. Les délibérations aboutirent à la création d'un groupement : Western Food and Game Fishes Protcclive Association qui se proposa, comme objectif immédiat, l'amendement de certains projets d'irrigation suscitant, du point de vue piscicole, le sérieuses appréhensions.

Des délégués se rendirent aussitòt à Washingon et réussirent à obtenir du $70^{e}$ Congrès, alors en session, le vote de la loi ci-après : ( 1 n act lo provide for the Conservalion of Fish and other pourposes).

"Il est décidé par le Sénat et la Chambre des Représentunts des Etatsl'nis d'Amérique, assemblés en Congrès, que le ministère du Commerce est, par les présentes, autorisé à étudier, rechercher et déterminer les meilleurs moyens ou procédés pour prévenir la destruction du Poisson occasionnée par les dérivations, canaux et autres ouvrages construits ou entretenus par les Ftats-Unis ; à cette fin sont autorisées, par les présentes, les dépenses nécessaires jusqu’à un montant de $2 \overline{5}$.owo (lollars (6.37.500 fr.) a prélever sur les ressources du Trésor n asant pas reçu d'autre affectalion ").

M. Shirley Baker, Ingénieur Conseil à San Francisco, fut alors préposé aux recherches par le Bureau of Fisheries, et prit comme collaborateur iI. U. B. Gilnoy, Ingénieur civil.

Le 20 Juin I 998, M. Henry O’Malckx, directeur du Service fédéral des pêches, présidait à Portland (Oregon) une réunion de tous les intéressés pour arrèter le programme des investigations.

Durant les cinq mois qui suivirent, les experts procédèrent sur place à l'examen de tous les types de grilles (1) préalablement essayés, prirent connaissance des nombreux projets ou modèles qui avaient été soumis aux livers services officiels, consultèrent enfin toute la littérature sur la matière.

Le 3o Novembre i929, MII. Baker et Gilrov déposèrent un rapport préliminaire et le 22 Janvier rg3o lcur rapport d'ensemble pour la période 1928 - 1929 (2).

Un voit que leur activité fut digne d'ólogres.

La conclusion formulée au terme de l'enquète est que, pour toutes les

(1) L'enruçte porta également sur les échelles ou élévaleurs à Poissons et ahoutit atux

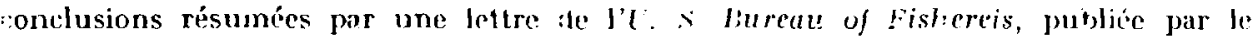
lsulletin d'Aoùt 1930 , p. 41 .

(2) Nous devons à l'obligeance de M. OMarux, Commissioner of Fisheries, la communication de ces deux documents et le prions de trouver ici lexpression de notre ineilleure gratitude. 
canalisations, agricoles ou industrielles, on peut maintenant s'opposer au dévalage des Poissons, de manière efficace et économique, sans entraver l'écoulement de l'eau. Lorsque ces canalisations sont de médiocre largeur irrigation), on utilise les disposilifs mécanicjues d'arrèt (mechanical fish screens) ; quand elles ont un débit relativement important (force motrice), on a recours aux dispositifs électruyues (olectric fish screens).

Ie mieux est, dans tous les cas, d'intercepter la descente du Poisson à i origine mème des dérivations, ce qui dispense d'avoir à barrer leurs ramifications par de multiples owrages.

Ce principe général posé, voyons comment, pour l'un et l'autre procédé, sont actuellement mises an point les installations slandard.

\section{I. - Ippareils mécaniques}

Après avoir examiné une dizaine de prises d'eau pour l'hydraulique agricole dans les Etats de Montana, Oregon et Washington, MM. Baker et Girnor furent amenés à condamner sans appel toutes les grilles fixes, soit horizontales, soit verticales, avee ou sans vannettes. Quel que soit leur agencement, elles s'obstruent toujours plus ou moins vite et, par suite, ne sont pas pratiques.

Le seul moyen d'óviter cet inconvénient grave est lemploi de grillages tournants (revolving screens), constitués par un tambour en treillis métallique, mobile autour d'un axe horizontal, qui, battant à contresens le courant à l'origrine de la dérivation, fait rebrousser chemin aux Poissons qui le viennent heurter. Par contre, les feuilles, herbes et autres débris charriés, sont entrainés par le cylindre dans son mouvement de rotation et transbordés d’amont en aval. Le flot, après les avoir plaqués contre les mailles au début de leur course circulaire, les détache au moment où elle s'achève ; ainsi le nettoyagre est-il automatique ; l'écoulement de l'eau n'est jamais entravé.

Des observations faites par les experts sur les diverses installations visitées, il se déduit que l'efficacité du système est subordonnée aux conditions suivantes :

I. - L'appareil doit être suffisamment robuste, convenablement logé et mû par le courant de la dérivation cqu'il intercepte ;

2. - Aucun jeu n'est admissible entre le grillage mobile et le fond ou les parois de son coursier;

3. - Les mailles seront assez fines pour retenir les Poissons les plus petits ;

4. - Une herse rustique est requise, à l'amont du tambour, pour le préserver du choc des corps flottants ou en suspension de quelque importance ;

5. - Ine rigole de raccordement bien tracée reliera la tête de l'installation à la rivière fournissant l'eau, pour que puissent la regagner aisément les Poissons arrêtés par l'obstacle.

En formulant ces règles fondamentales, les techniciens de l'U. $S$. Bureau of Fisheries n'ont fait, comme ils le signalent eux-mêmes, que dégager, 
en les précisant, celles suivies depuis i 92 I par l'Oregon Game Commission. I l'époque où commença leur mission, il y avait sept ans déjà qu'avaient été imaginés et mis en service, par l'organisme en question, les premiers mechanical fish screens. Des perfectionnements successifs furent, par la suite, apportés aux appareils, dont le modèle le plus recommandable était, au témoignage de MM. Baken et GiLrox, celui de Savage Rapids Dam (Grants Pass Irrigation District). sur la Rogue river, fleuve côtier se jetant dans le Pacifique un peu au sud du Cap Blanco. Construit en 1924, moyennant une dépense de 300 dollars ( 6.500 fr.) environ, le modèle en question avait donné et donnait encore toute satisfaction.

Presque en môme temps que dans l'Orégon le système du barrage mobile fut adopté dans l'Etat voisin de Washington, sur l'initiative de M. C.-A. Coвb, Surintendant de la Yakima Valley Water Company, auquel on doit l'utile amélioration que constitue le canal de raccordement, grâce auquel les Poissons arrêtés dans leur descente ont toutes facilités pour retourner au cours d'eau d'où ils viennent.

Ces brèves indications données sur la genèse des grillages tournants, on sera complètement renseigné sur leur compte par la description d'une installation conforme aux principes posés par les spécialistes.

La plus moderne est celle du canal Ahtanum (bassin de la Columbia), dans la Réserve indienne de Yakima, à 27 kilomètres environ au sud de la localité de même nom (Etat de Washington). Le projet, établi par MM. Barer et Gilroy, avec la collaboration de M. W.-R. Coloman, Surintendant de l'Oregon Game Commission, a été, en effet, exécuté au début de 1930 .

Le volume d'eau dérivé est sujet à très larges fluctuations, car s'il atteint 2.225 litres-seconde au commencement de la saison d'irrigation, il tombe à 140 l. -5 . vers la fin.

Dans ces conditions, et en prévision d une extension ultérieure des surfaces à abreuver, on a placé, côte à côte, deux appareils identiques. Sclon les besoins on met en marche un seul d'entre eux ou la paire.

Les circonstances topographiques ont amené à placer les mechanical screens à 3oo mètres au-dessous de la prise d'eau dans l'Ahtanum creeck, sur un canal d'adduction de 4 mètres de largeur moyenne et I $\mathrm{m}$. 20 de profondeur maxima.

Lc plan et la photogravure ci-après (Fig. is et rg) explicitent les détails de l'installation. Il suffit, ce semble, de compléter l'information visuelle par quelques précisions ct données numériques.

Les dimensions extrêmes de l'ensemble de la construction sont : dans le sens du courant : 7 M. 32 ; transversalement : 7 M. 72 ; en élévation : I M. 22. Le coursier a, au total, \& M. 88 de largeur, mais une murette le divise, longitudinalement, en deux couloirs semblables, ayant 2 M. 34 entre parois.

Sur la gauche, à go centimètres de la tête de l'ouvrage, s'échancre, sur coute la hauteur, l'ouverture rectangulaire de la rigole de raccordement, large de $45 \mathrm{~cm}$. 


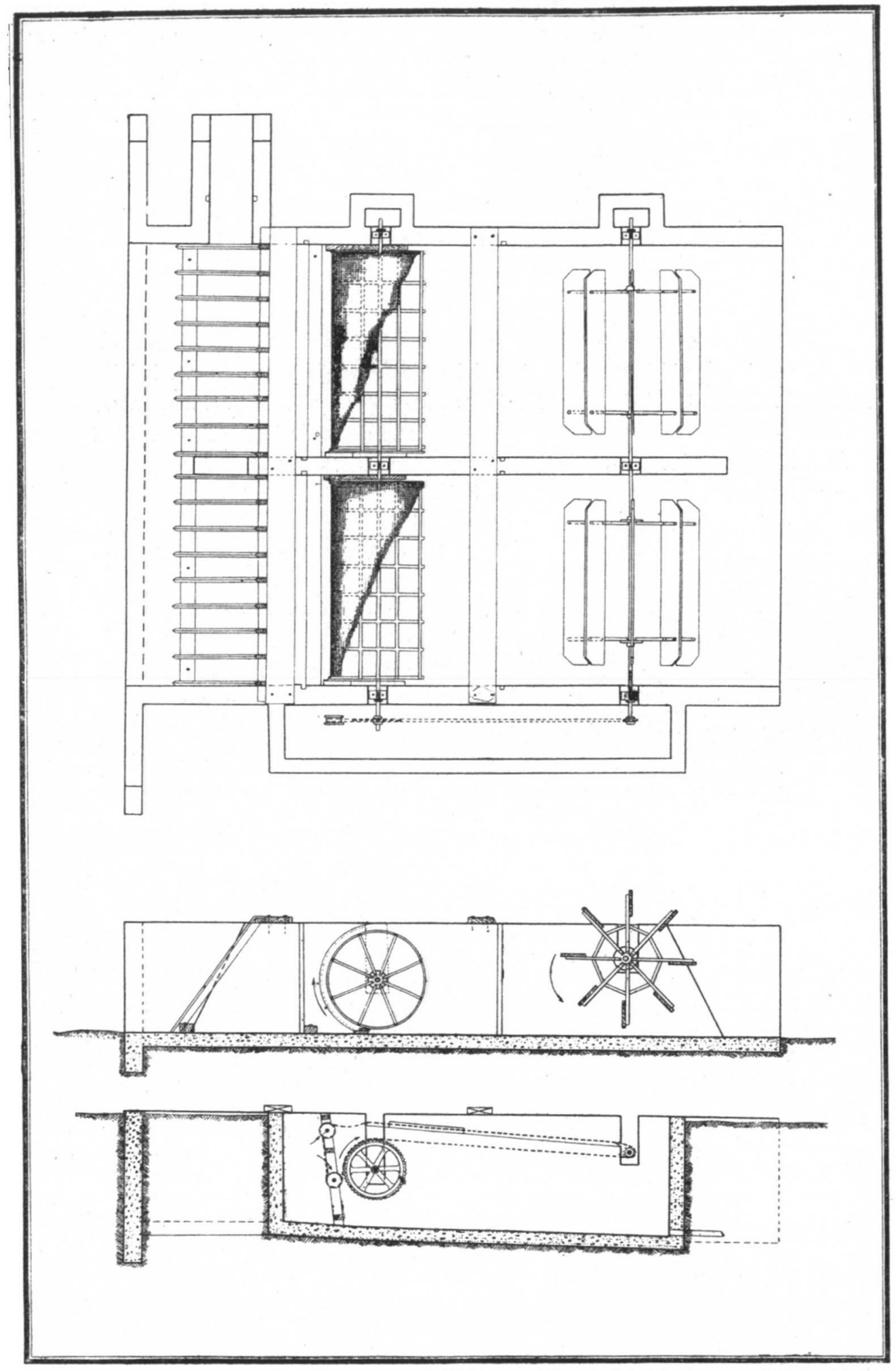

Fig. 18. - Plan et coupes du grillage tournant d'Ahtanum (Etat de Washington). - Echelle : 1/24,4. 
Au flanc droit s accole la fosse des transmissions, dont le vide intérieur mesure, en plan, 4 Y. 35 sur $6 \mathbf{r} \mathrm{cm}$.

L'épaisseur du béton est de rá centimètres pour le radier et les parois de la fosse latérale, de 20 centimètres pour toules autres parties en élévation.

Des rainures verticales, de $\bar{j} \mathbf{~} \mathbf{m}$. sur $\bar{j} \mathbf{c m}$, à l'origrine de la rigole de raccordement et en avant des grillages tournants et des roues à palettes les actionnant forment les golissières de vannettes servant à arrêter l'eau ou à en régler le débit.

Devant chacun des couloirs s'interprose unc herse inclinée à $53^{\circ}$ sur l'ho-

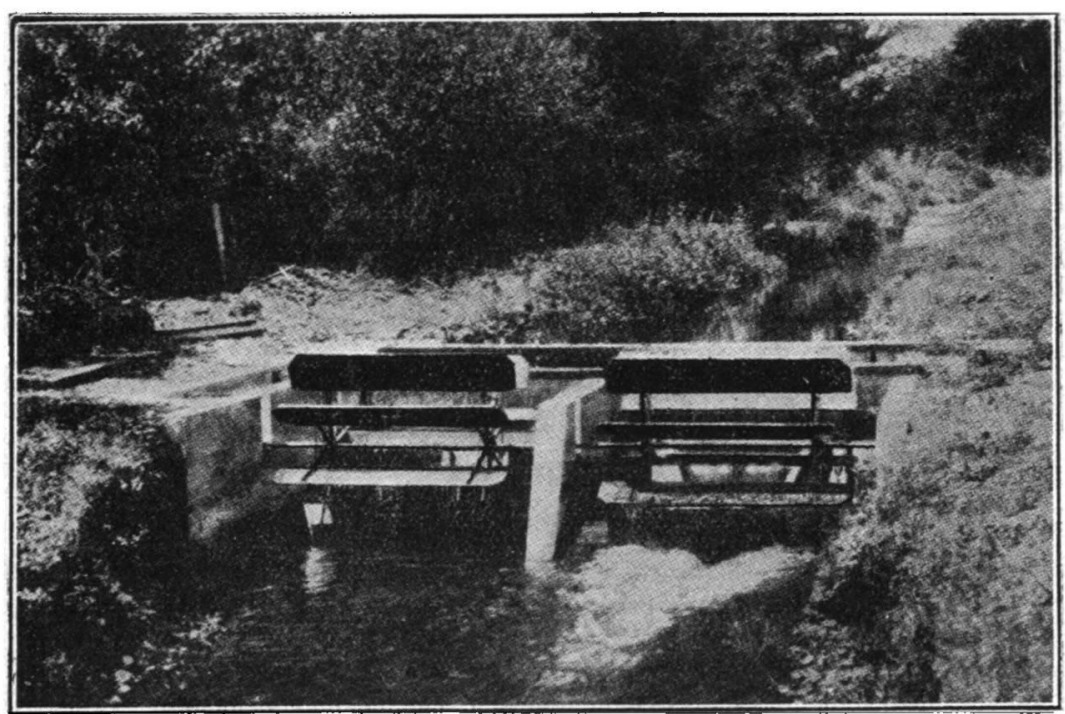

Fic, 19. - Grillages tournants didhtanum. - Vue diatial (1).

rizontale ; clle est forméc de g barreaux de bois de $5 \mathrm{~cm}$. sur so $\mathrm{cm}$., espacés de $23 \mathrm{~cm}$. I/2, s'assemblant dans une tête de mème écquarrissage et une :cmelle de $10 \mathrm{~cm}$. sur $15 \mathrm{~cm}$; ; res deux traverses sont situées respectivezuent, à peu de chose près, dans lo prolongement des murettes aval of amont de la rigole de raccordement.

A 2 M. zo environ de l'entréc du coursier une entaille dans les parois et la cloison séparative, Jarge de $20 \mathrm{~cm}$., profonde de $9 \% \mathrm{~cm}$., marque l'emplacement de l'axe en fer rond de 27 millimètres des yrillages mobiles. Char un d'eux est un tambour de 2. 11 . I/ de longueur et $53 \mathrm{~cm}$. de rayon, dont la carcasse en fer plat de $2.5 \mathrm{~mm}$. sur $6 \mathrm{nim}$., est constituéc par huit cercles équidistants soutenus chacun par 8 rayons branchés sur l'arbre, et entretoisés par des tringles reliant leurs tèles qui forment génératrices du cylindre. Cette armature est revêue d'un treillis de fil do fer galvanisé à mailles de $8 \mathrm{~mm}, \mathrm{r} / \mathrm{h}$.

(1) D'après une illustration des Propagalion and Distribution of Food Fishes 1930. Annexe 16 au Report of Commeissioner of Fisherios for the fiscal year 1930. Washington, $\mathrm{r} 93 \mathrm{r}$. 
Sur le radier, très peu en amont de l'aplomb de l'axe, est fixée urra planche de $20 \mathrm{~cm}$. de largeur sur $5 \mathrm{~cm}$. d'épaisseur, au rebord de laquelle est clouée une bande de gros caoutchouc qui, rebroussée par le tambour dans son mowrement de rotation, reste en contact intime avec lui. De même, sur toute la hauteur des parois du couloir, à l'emplacement de l'appareil, sont scellés des panncaux en bois de $30 \mathrm{~cm}$. de largeur sur $5 \mathrm{~cm}$. d'épaisseur, sur lesquels est fixée une garniture semi-circulaire, similaire de la précédente, découpée dans un vicux handage pneumatique, qui épouse le contour des cercles extrèmes. De la sorle, latéralement comme sur le fond, toute issue est fermée au l'oisson se laissant aller au fil de l'eau.

l ? M. 82 de l'axe du tambour se trouve celui de la roue à aubeş qui l'actionne, au fond d'une échancrure du béton avant $20 \mathrm{~cm}$. de largeur ct $53 \mathrm{~cm}$. I $/ 2$ de profondeur. Cette roue a I M. 83 de longueur et $76 \mathrm{~cm}$. de rạon, ce qui laisse un vide de $7 \mathrm{~cm}$. $1 / 2$ entre elle et le radier. Le bâti est unélallique; l'arbre, de $27 \mathrm{~mm}$. de diamètre, porte deux couronnes de 8 rayons entretoisés, en fer cornière de $3 \mathrm{I} \mathrm{mm}$. sur $4 \mathrm{~mm}$. I/2. Distantes de I M. 22 , clles soutiennent les palettes en bois de pin, larges de $30 \mathrm{~cm}$., ́paisses de $25 \mathrm{~mm}$., aux angles extérieurs abattus.

Les arbres de la roue et du tambour se prolongent dans la fosse des transmissions où sont calées, sur le premier un pignon à $g$ dents, sur le second une rour en portant 48. La liaison est assurée par l'intermédiaire d'une lonque chaîne à maillons. Pour obtenir les rotations en sens contraire des deux axes, cette chaîne, passant sur deux poulies de renvoi placées in avant de la roue dentée, embraye à sa partie supérieure sur un quart de la rirconférence, entraînant la jante dans le sens des aiguilles d'une montre.

Le type qui vient d'être décrit comporte des variantes ; c'est ainsi, par exemple, qu'une roue à augets preut être substituée à la roue à aubes, comme à Savage Rapids Dam. Il faut adapter le dispositif aux conditions locales, mais à la condition de satisfaire aux conditions fondamentales posóes par MY. Baker et Girroy, on a la certitude d'un fonctionnement pleincment satisfaisant. L'expérience des dix dernières années faif voir dans le mechanical fish screen, lancé parl'Oregon Game Commission, perfertionné par M. C.-A. Cons, une solution excellente, pour les petites ou moyennes rị̣oles, du problème consistant à retenir les Poissons qui dévalont sans entraver l'écoulement de l'cau.

En France, les grillages tournants rendront les mêmes scrvices qu'en Imérique, si on en installe à l'origine des dérivations servant à l'irrigation les prairies : on sait qur ces dernières sont de redoutables pièges à tacons - f truitclles. Peut être cerfains salmonicultcurs auront-ils aussi intérêt, pour assurer à leurs viviers une alimentation régulière en cau, à remplacer par ces appareils des clayettes fixes dont l'obstruction est si fréquente, le nettoyage si fastidieux et coutcux. Ils ont là, par surcroît, un moven de s'opposer à l'évasion de leurs pensionnaires qui, à l'occasion, sautent d'un bond viģoureux des pièces d'eau dans les canaux d'évacuation.

(A suivre). 